\title{
Design of a Configurable Remote Data Acquisition System
}

\author{
Jiawen $\mathrm{He}^{1, a}$, Jiqiang $\mathrm{Xia}^{2, \mathrm{~b}}$ \\ ${ }^{1}$ School of Mechanical Engineering \& Automation, Beihang University, Beijing, P.R. China \\ ${ }^{2}$ School of Mechanical Engineering \& Automation, Beihang University, Beijing, P.R. China \\ ahejiawen1993@163.com, bxiajiqiang@buaa.edu.cn
}

\begin{abstract}
Keywords: configurable, remote access, data acquisition, LabVIEW
Abstract. With the development of information technology, especially computer networks, remote acquisition based on the Internet has become a new trend of measurement and control systems. In this paper, a versatile and configurable data acquisition system for measuring engine parameters is presented using the integration of an instrumentation software package, LabVIEW and a relational database, MySQL. Supported by techniques of database, internet and real-time operating system, network communication module, data acquisition module, graphic monitoring module and data management module are integrated into the system. In addition, to acquire various types of transducer's signals from a variable number of channels, a system configuration module is developed that makes it possible for operators to customize the system. Finally, the practicability and validity are verified with an instance.
\end{abstract}

\section{Introduction}

The technology advancement and the wide use of the computer help measurement systems play an essential role in many of the scientific as well as everyday applications [1], which overcome the inconvenience of physical measurement systems that an operator manually carries out the procedure. Benifiting from the Internet, distributive measurement systems where instruments are connected to a server that communicates with the client over the network provide solutions for acquiring field test data remotely[2].

In the engine test, the data acquisition requests high real-time and synchronization while the operator needs a friendly human-computer interaction interface. As a result, the client and the data acquisition device present different requirements. A remote data acquisition system with an industrial control computer for data acquisition and a desktop for client may be best suited for the needs. At present, common data acquisition systems are often dedicated acquisition systems for specific objects and specialized applications[3], making it relatively laborious in terms of system configurability, scalability and maintainability [6]. To solve this problem, the data acquisition system designed in this paper is integrated with system configuration function. With the system configuration module, operators can configure the system according to their needs, such as channel configuration, display parameter configuration, which makes the system somehow versatile and can be easily maintained, applied and extended.

In order to meet the requirements of the test bench for data acquisition with better flexibility, this article introduces NI PXI acquisition hardware and a systems engineering software, LabVIEW to implement remote system configuration, data acquisition, remote data transmission, data real-time display and data management.

\section{System Design and Architecture}

In this paper, the configurable remote data acquisition system adopts a client/sever architecture which consists of a data acquisition computer as the server and a desktop as the client. by dividing functions of sharing information into the client and server, the C/S architecture is applied to both large- and small-scale applications [7]. Users can select the operating system and develop the 
corresponding software as required. The data acquisition is handled by the server, and the results are returned to the clients

Software Architecture. In this paper, the server comes loaded with an industrial real-time operating system called Phar Lap ETS that is based on task priority[7]. The server software is composed of a command parsing module, a data acquisition module with high priority and a data sending module with medium priority. The client is mainly composed of a system configuration module, a command sending module, a data receiving module and a user interface. The system block diagram refers to Fig. 1:

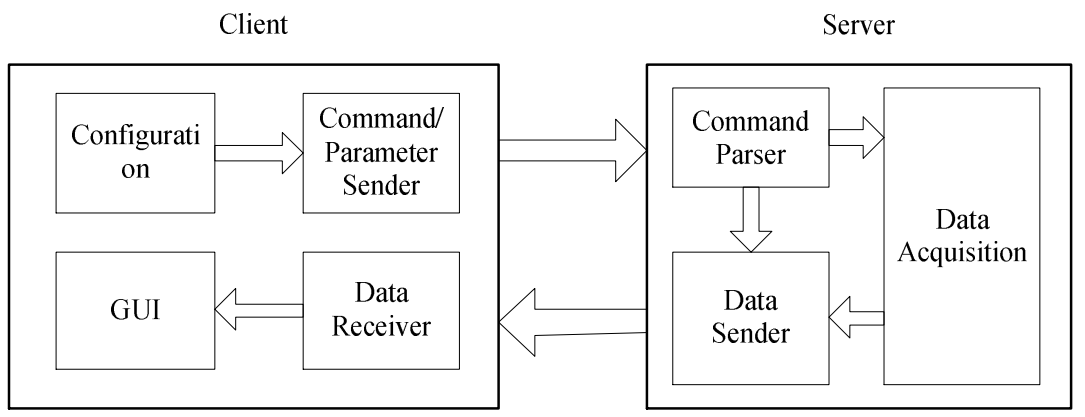

Fig. 1 Architecture of the system

Hardware. As for the hardware, the server that act as a data acquisition terminal is equiped with a PXI embedded controller and NI data acquisition boards. The embedded controller offers a high-performance plat form for measurement applications. PXI-6251 with SCXI terminal blocks plug into the controller for colleting analog signals, and PXI-6528 for digital signals, PXI-6624 for counter/timer signals. The client is actually a desktop with client software developed with LabVIEW. Data is transmitted via TCP/IP. The hardware diagram refers to Fig. 2:

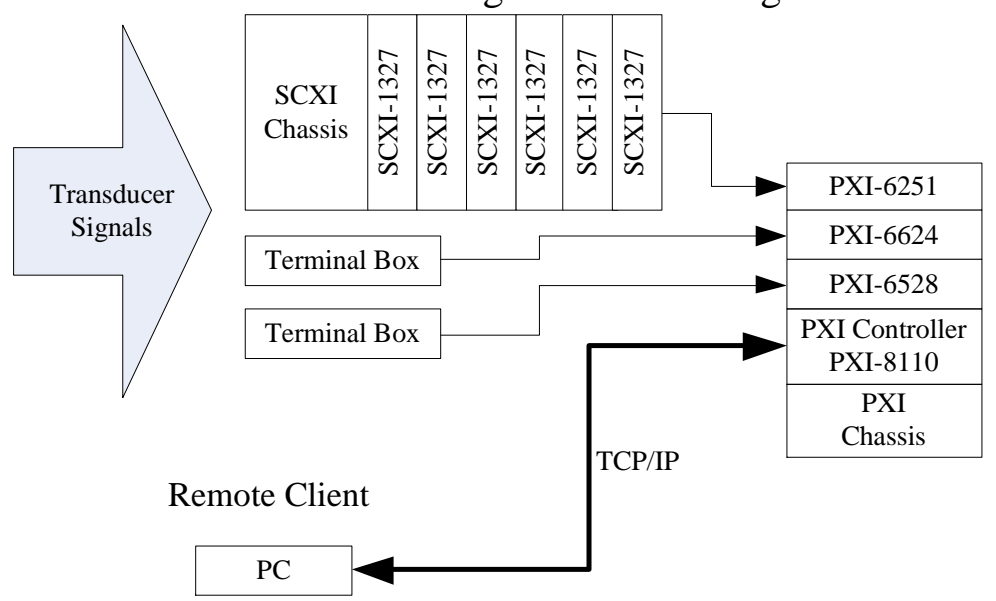

Fig. 2 Hardware of the system

\section{Software Design}

The software of this paper is developed using LabVIEW from National Instruments (NI). Both the client software and the server software are multithread applications where different processes are executed concurrently. They are introduced in the following.

System Configuration. To make the system more flexible as well as to take advantages of distributed data acquisition and the PXI series hardware, the system configuration module is designed to get remote access to configuring the system parameters. The operator can easily set the server-side acquisition program on the client with the module when there is a change in acquisition requirements or the acquisition hardware. The implementation of the remote configuration module ensures that the system can work with various types of signals, acquisition parameters and hardware devices.

The remote configuration of the system can be accomplished following these steps. Firstly, the information of all hardware devices connected to the server is acquired. Then the most recently used 
configuration file is loaded and the operator shall be informed if the loaded configuration does not match the current set of hardware. The operator can change the configuration or create a new one to meet the test needs or to match the connected hardware. Finally, the configuration is saved as a new file so that different configuration files can be transmitted to the server through the Internet when different acquisition tasks are carried out.

Data Acquisition. Data acquisition that is the core function of this system can collect analog signals, digital signals and counter signals. In other words, this data acquisition system can meet the requirements of all tasks the test stand may carry out. The process of real-time data acquisition refers to Fig. 3:

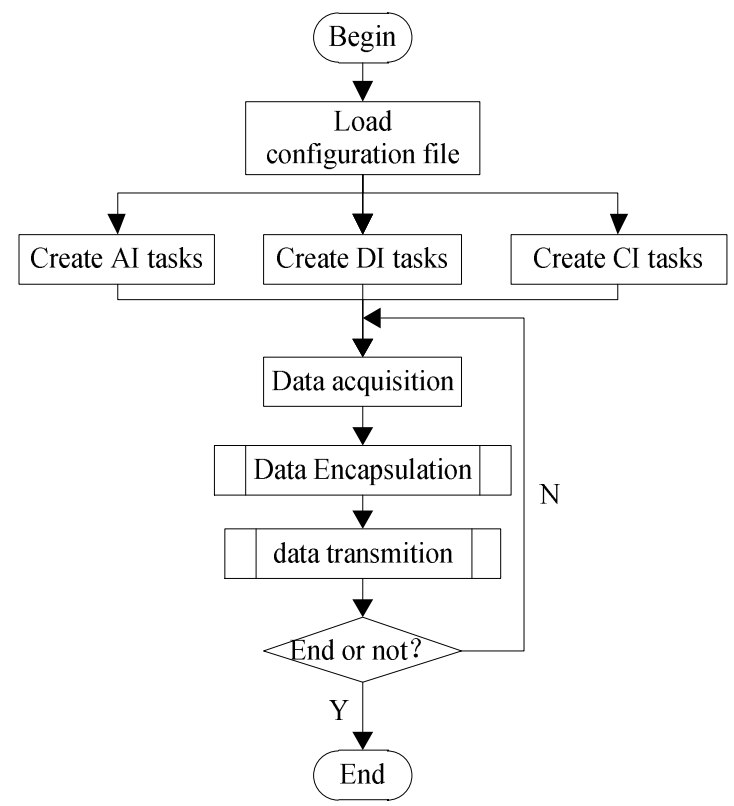

Fig. 3 Flow chart of data acquisition module

After the system is initialized, the configuration file is loaded first, and corresponding tasks are created based on the configuration file. Next, the loop of the acquisition task is executed which includes data acquisition, data encapsulation and data transmission. In order to improve the real-time performance, multithreaded programming is adopted to separate the data encapsulation and data transmission from the acquisition loop by creating child processes. By setting the priority of the data collection task to be high and the priority of other tasks to be medium, the real-time performance is guaranteed by the real-time operating system scheduling which is based on the priority.

Network Communication and Data Transmission. The system in this paper follows a client/server model and data is transmitted via TCP/IP protocol. The Internet protocol suite (commonly TCP/IP) is the set of communications protocols that implement protocol stack on which the Internet and most commercial networks run [9]. LabVIEW provides a built-in TCP/IP network communication suite that makes it convenient to communicate using TCP by invoking APIs one of which is called Simple Messaging. There is no need to worry about the underlying implementation of the network, thereby freeing the engineer from the network details.

The data acquisition system of this paper adopts the client/server communication mode in design. The system is divided into two parts: The processing host works in client mode whose jobs are the data reception and providing an interface for follow-up data processing. While the data acquisition stand works in server mode to transfer data.

To ensure a smooth transfer of data, data packets need to be encapsulated. Using formatted packets makes data more manageable and easier to parse. Different types of packets such as configuration, command and data are distinguished by the Data ID. The packet encapsulation format is shown as follows:

\begin{tabular}{|c|c|c|}
\hline $\begin{array}{c}\text { Data Size } \\
(32 \text { bits })\end{array}$ & $\begin{array}{c}\text { Data ID } \\
(16 \text { bits })\end{array}$ & Data \\
\hline
\end{tabular}


In this paper, the server is permanently running and waiting for connections. Once a connection is established, all Data IDs are sent to the network to complete the initialization. Then data packets encapsulated according to the Data ID and sent to the network. Referring to Fig. 4 (a):

In the client software, a TCP connection of the specified IP address and port is established first. Then use the STM Read function to receive the data packets, and parse the packets according to the specified Data ID. The parsed data is then transmitted to data processing program for further processing and displaying. Referring to Fig. 4 (b):

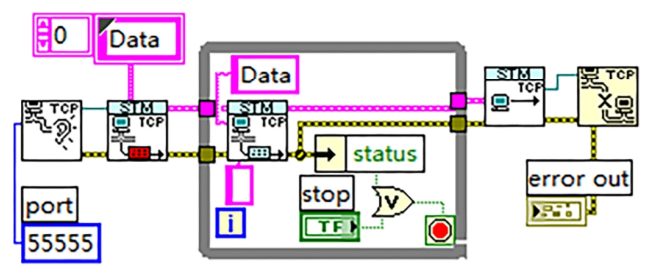

(a)

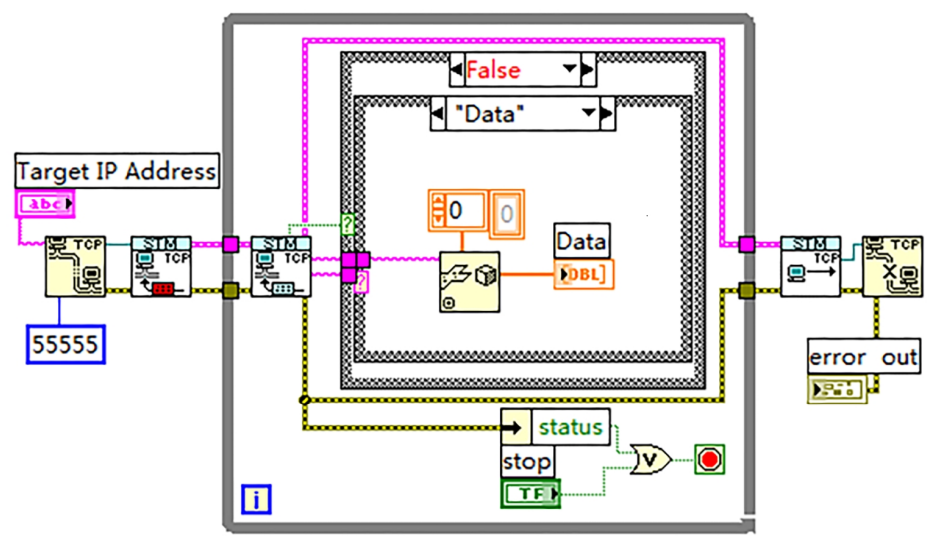

(b)

Fig. 4 Network communication of server and client

User Interface and Data Management. On the client-side, user interface is designed for system operation and real-time data display so that operators can process and manage data received. All display controls can be customized. The operator can select the data to be displayed and configure the display mode ( numerical or waveform). As for data management, all data is stored in the database which facilitates processing and analysis of data after testing. At the same time, the operator can record the data that he is interested in, such as certain steady-state parameters, at any time during the test to generate a test report. Besides, data playback function is integrated in the system. The operator can view the historical data for detailed analysis and diagnosis of the tesed unit.

\section{Case Study}

In order to verify the correctness of the data acquisition system, this paper has been configured for a certain aircraft auxiliary engine test stand to acquire and manage data. Parameters such as engine speed, power, pressure, temperature and flow rate are acquired by the server and transmitted to the client over the network to display, store and manage.

After initialization and configuration, the physical channel, signal type, input range and conversion relation of related signals are configured. The configuration file of current test is transferred to the sever-side. Then the channels displayed by and the alarm thresholds of each control are set. Finally, data acquisition begins and the system works, as is shown in Fig. 5.

\section{Conclusions}

Based on the research of virtual instrument and data acquisition technology, this paper presents a configurable remote data acquisition system based on LabVIEW development platform and hardware from National Instruments. To some extent, this system solve the problems that common remote data acquisition systems are dedicated for specific objects and specialized applications by provide remote access to configuring the system. Besides, by adopting client/server architecture where data acquisition is handled by the server with real-time operating system and users operate on the client, the system performance and maintainability are enhanced. Verified by experiments, the modules like remote configuration, data acquisition, transmission and management works well. In conclusion, the system is practical in data acquisition. 


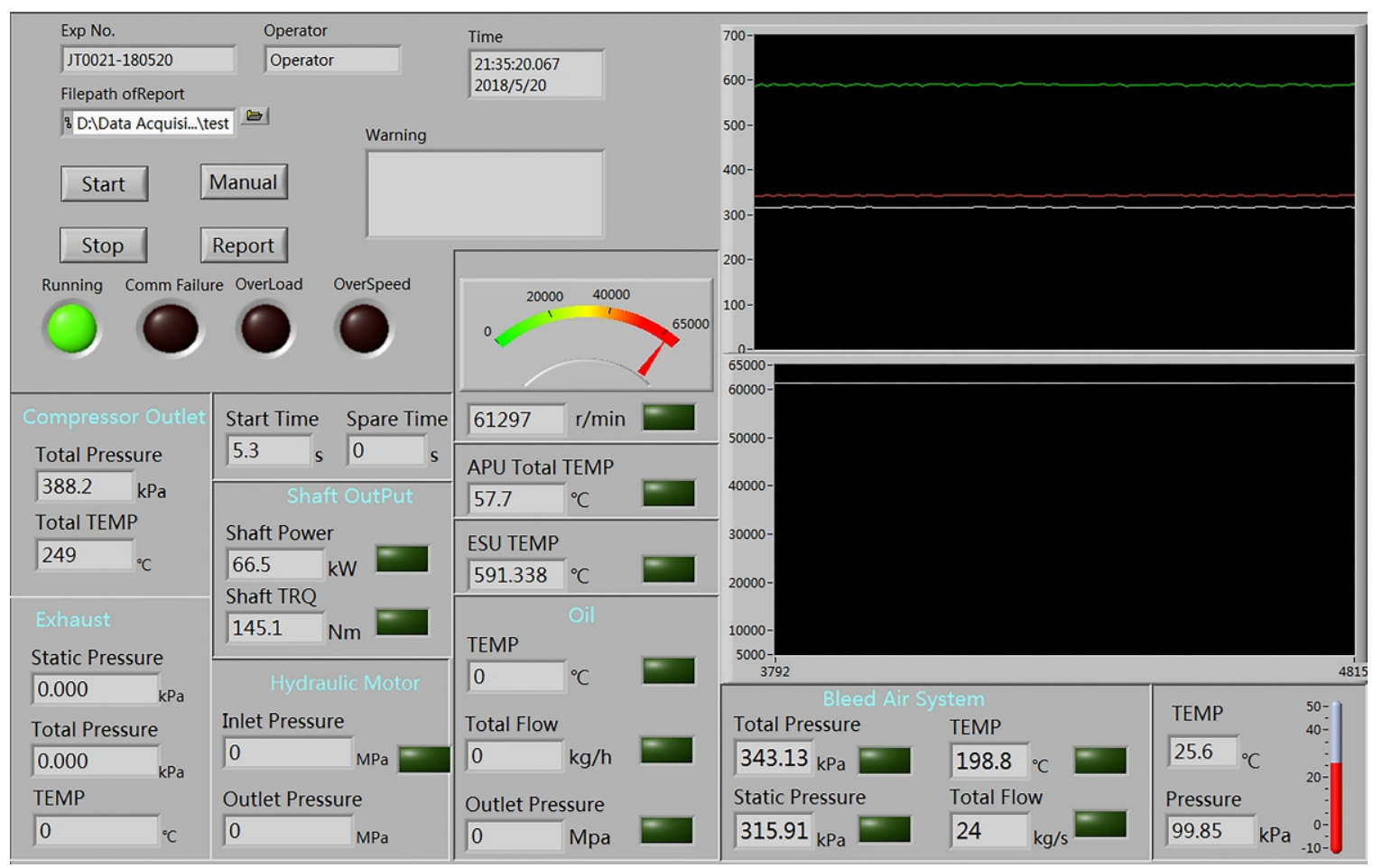

Fig. 5 Illustration of system performance

\section{References}

[1] S. Ji, Y. Lei and W. Zhang. The design of data acquisition system based on virtual instrument. 2012 2nd International Conference on Computer Science and Network Technology (ICCSNT). Changchun, 2012, p. 594-597.

[2] M. Abdelrahman and A. Rasheed. A methodology for development of configurable remote access measurement system. Isa Trans. Vol.39 (2000) No.4, p. 441-458.

[3] C. Zheng, M. Dai, Z. Zhang, Y. Hu and Y. Guo. Real-time remote data acquisition and process monitoring system for automatic filling line. 2017 24th International Conference on Mechatronics and Machine Vision in Practice (M2VIP). Auckland, 2017, p. 1-5.

[4] X. Zhang and C.Hu. The Development of Data Acquisition and Remote Real-Time Display System;for EAST NBI. Journal of Fusion Energy. Vol.32 (2013) No.5, p. 566-569.

[5] J. Du, W. Li and J. Guo. "Design of LabVIEW based general data acquisition system. 2017 IEEE 2nd Information Technology, Networking, Electronic and Automation Control Conference (ITNEC). Chengdu, 2017, p. 1235-1239

[6] RL.Shuler, Auto-routable, configurable, daisy chainable data acquisition system. United States Patent No.6943621 B1 (2005).

[7] HS. Oluwatosin. Client-Server Model. IOSR Journal of Computer Engineering (IOSR-JCE). Vol.16 (2014) No.1, p. 57-71.

[8] P. Podešva and D. Fojtík, Creation of control applications for operating system Phar Lap, 2011 12th International Carpathian Control Conference (ICCC). Velke Karlovice, 2011, p. 312-315.

[9] DG. Zutin,ME Auer,JF Bocanegra et al. TCP/IP Communication between Server and Client in Multi User Remote Lab Applications. International Journal of Online Engineering. Vol.4 (2008) No.3, p. 42-45. 